九州大学学術情報リポジトリ

Kyushu University Institutional Repository

\title{
Nonlinear evolution of electromagnetic waves driven by the relativistic ring distribution
}

Matsukiyo, Shuichi

Department of Earth System Science and Technology, Kyushu University

Hada, Tohru

Department of Earth System Science and Technology, Kyushu University

http://hdl. handle. net/2324/16867

出版情報: Physics of Plasmas. 9 (2)，pp.649-661，2002-02. American Institute of Physics バージョン：

権利関係 : 


\title{
Nonlinear evolution of electromagnetic waves driven by the relativistic ring distribution
}

\author{
S. Matsukiyo ${ }^{\text {a) }}$ and T. Hada \\ Department of Earth System Science and Technology, Kyushu University, Fukuoka, 816-8580, Japan
}

(Received 9 July 2001; accepted 7 November 2001)

\begin{abstract}
Relativistic ring distribution of plasma particles generates electromagnetic waves via the relativistic cyclotron resonance. The long time evolution of this so-called cyclotron maser instability at null wave number $(k=0)$ is studied in detail, by performing particle simulations using a plasma which consists of relativistic ring electrons, background positrons, and background electrons. The linear and nonlinear stages of the system evolution are discussed for both gyrotropic and nongyrotropic ring distributions. The linear theory predicts that, when the initial ring energy is strongly relativistic, there appears a critical initial ring momentum at which the system is marginally stable. Numerical simulations show, however, that the system is nonlinearly unstable even when the initial ring momentum exceeds the critical momentum. The final saturation level of the wave energy is obtained analytically. ㅇ 2002 American Institute of Physics. [DOI: 10.1063/1.1431593]
\end{abstract}

\section{INTRODUCTION}

One of the eminent relativistic effects in a plasma is a downshift of the particle cyclotron frequency due to relativistic mass increase. Consequently, the resonance condition between particles and waves is modified by the Lorentz factor, $\gamma$,

$$
\omega-k_{\|} v_{\|}-\Omega_{0} / \gamma=0,
$$

where $\omega$ is the wave frequency, $k_{\|}$the parallel wave number, $v_{\|}$the parallel particle velocity, and $\Omega_{0}$ is the nonrelativistic cyclotron frequency, respectively. The Lorentz factor $\gamma$ $=\sqrt{1+\left(p_{\|}^{2}+p_{\perp}^{2}\right) / m_{0}^{2} c^{2}}$ depends both on parallel $\left(p_{\|}\right)$and perpendicular $\left(p_{\perp}\right)$ momenta, where $m_{0}$ is the particle rest mass and $c$ is the speed of light. The relativistic resonance condition represents an ellipse (for $\omega / k_{\|} c>1$ ) or a hyperbola (for $\omega / k_{\|}<<1$ ) in the $p_{\|}-p_{\perp}$ space, while the nonrelativistic one represents a straight line. This difference in the resonance conditions leads to some drastic changes in certain plasma instabilities.

The ring instability is an ideal subject to study such effects. The ring distribution generates electromagnetic waves via the cyclotron resonance, Eq. (1). When $\omega / k_{\|} c<1$, the well-known temperature anisotropy driven instability is excited in both nonrelativistic $(\gamma=1)$ and relativistic $(\gamma>1)$ plasmas. However, if $\omega / k_{\|}>1$, only the waves satisfying the relativistic resonance condition can interact with the ring distribution. Such waves are called the cyclotron maser emission. A review on the kinetic cyclotron maser instability is given by Wu. ${ }^{1}$ The cyclotron maser instability has been discussed extensively, for applications in space plasma (e.g., the auroral kilometric radiation, or the $\mathrm{AKR}^{2}$ ) and for laboratory experiments. ${ }^{3}$

The mechanism of the electromagnetic radiation due to the cyclotron maser instability was proposed in the late 1950 's, independently by Twiss, ${ }^{4}$ Schneider, ${ }^{5}$ and Gaponov. ${ }^{6}$

${ }^{a)}$ Electronic mail: matsukiy@esst.kyushu-u.ac.jp
The waves are generated as a result of azimuthal bunching of ring particles caused by the relativistic mass dependence of the particle cyclotron frequency. Chu and Hirshfield ${ }^{3}$ analyzed a linear dispersion relation of the cyclotron maser instability assuming the delta function type relativistic ring distribution as a free energy source. They compared two unstable modes: the temperature anisotropy driven instability $\left(\omega / k_{\|} c<1\right)$ and the cyclotron maser instability $\left(\omega / k_{\|} c\right.$ $>1$ ), for parallel propagation. One of the most successful applications of the cyclotron maser instability to space plasma phenomena was the theory of AKR proposed by $\mathrm{Wu}$ and Lee. ${ }^{2}$ They explained the generation of the X-mode waves observed in the AKR source region by using the relativistic loss-cone distribution. Galeev and Krasnosel'skikh ${ }^{7}$ also, around the same time, suggested that the synchrotron instability can generate the S-bursts in Jupiter radio emission. Winglee ${ }^{8}$ discussed in detail the linear growth rate of the cyclotron maser instability using a Dory-Guest-Harris (DGH) distribution function (Dory et al. ${ }^{9}$ ) including the finite temperature effect. It is concluded that the azimuthal bunching instability treated by $\mathrm{Chu}$ and Hirshfield ${ }^{3}$ is observed when the phase synchronization time scale is less than the wave growth time scale, and $\mathrm{Wu}$ and $\mathrm{Lee}^{2}$ instability is observed in the opposite case. Freund et al. ${ }^{10}$ studied multiple harmonics of the cyclotron resonance for oblique propagation.

The nonlinear stage of the instability has also been discussed theoretically and numerically (e.g., Queau, ${ }^{11}$ Mourenas et al. ${ }^{12}$ Pritchett, ${ }^{13}$ and Queau et al. ${ }^{14}$ ). According to these studies, there are two candidates for the wave saturation. When the excited waves have large frequency bandwidth, the quasi-linear diffusion stops the wave growth. On the other hand, when the waves have narrow frequency band width, the instability is mainly terminated by the particle trapping in the momentum space. The saturation levels for each mechanisms are obtained by the conditions of 
$\partial f_{0} / \partial p_{\perp}=0$ for the quasi-linear diffusion and $\delta_{l} \sim \Omega_{\text {trap }}$ for the trapping, respectively, where $\delta_{l}$ is the linear growth rate and $\Omega_{\text {trap }}$ is the trapping frequency of the ring particles.

For astrophysical applications, Volokitin et al. ${ }^{15}$ derived the linear dispersion relation and the quasi-linear evolution equation for the synchrotron instability in a relativistic electron-positron plasma in the pulsar magnetosphere. Hoshino and Arons ${ }^{16}$ performed numerical simulations of the instability assuming the homogeneous cold relativistic ring distributions of electrons and positrons, and studied the relaxation of the system. They compared the results with simulations by Langdon et al., ${ }^{17}$ Gallant et al. ${ }^{18}$ and Hoshino et al. ${ }^{19}$ who studied the structure of relativistic perpendicular shocks in electron-positron and electron-positronproton plasmas. It is concluded that the synchrotron maser instability efficiently thermalizes the particle distribution downstream of the shock.

Most of the previous studies focus on the parameter regime, $k_{\perp} c / \Omega_{0} \sim 1$, and the wave propagation direction around $90^{\circ}$ with respect to the ambient magnetic field. This is because the relativisitic cyclotron maser instability is expected to generate waves with a peak intensity around the parameters above. On the other hand, one of the most curious properties of this instability is that waves at null wave number $k=0$ can be destabilized, i.e., the instability without spatial dependence. Strictly speaking, there are always boundaries in realistic systems, so the $k=0$ mode cannot be allowed to exist, but consideration of this wave enables us to discuss nonlinear evolution of the system with high accuracy, since consideration of spatial dependence of the instability can be totally neglected. Furthermore, it makes the analytical treatment of strongly relativistic phenomena feasible, while most of the past studies only looked at weakly relativistic plasmas. In addition, we will discuss the effects of nongyrotropy of the ring distribution function.

\section{LINEAR THEORY}

\section{A. Dispersion relation for gyrotropic ring distribution}

We assume that the plasma consists of three components, background electrons, background positrons, and ring electrons. In this section, for simplicity, all the components are assumed to have no thermal spread. Then the distribution function of each component is written as

$$
\begin{aligned}
f_{j 0} & =\frac{n_{j 0}}{2 \pi p_{\perp}} \delta\left(p_{\|}\right) \delta\left(p_{\perp}\right), \\
f_{r 0} & =\frac{n_{r 0}}{2 \pi p_{\perp}} \delta\left(p_{\|}\right) \delta\left(p_{\perp}-p_{0}\right),
\end{aligned}
$$

where the subscripts $j$ and $r$ represent the plasma species $(j$ is the background electrons and positrons, and $r$ is the ring electrons), $n_{0}$ denotes the plasma density, $p_{\perp}$ and $p_{\|}$are the perpendicular and parallel momenta, and $p_{0}$ indicates the initial ring momentum, respectively.

The electromagnetic dispersion relation for parallel propagation is then given as

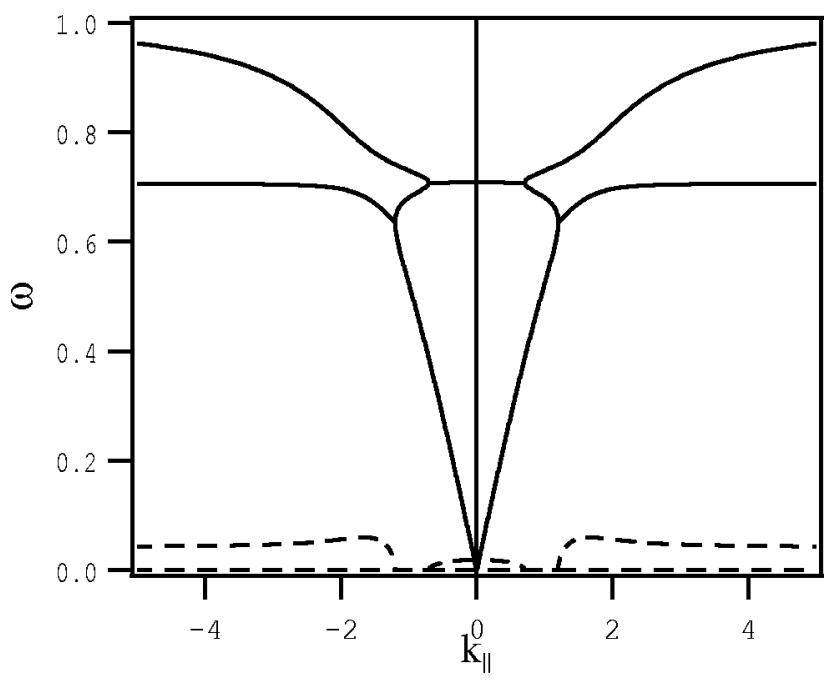

FIG. 1. Parallel dispersion relation of the electromagnetic waves [the solution of Eq. (4)] for $\alpha=0.01, a=p_{0}=1$. The solid and dashed lines show the real and the imaginary frequencies.

$$
\begin{aligned}
0= & -\frac{k_{\|}^{2} c^{2}}{\omega^{2}}-\frac{\omega_{p}^{2}}{\omega\left(\omega+\Omega_{0}\right)}-\frac{(1-\alpha) \omega_{p}^{2}}{\omega\left(\omega-\Omega_{0}\right)} \\
& -\frac{\alpha \omega_{p}^{2}}{\gamma_{0} \omega^{2}}\left(\frac{\gamma_{0} \omega}{\gamma_{0} \omega-\Omega_{0}}-\frac{p_{0}^{2}\left(\omega^{2}-k_{\|}^{2} c^{2}\right)}{2 m_{0}^{2} c^{2}\left(\gamma_{0} \omega-\Omega_{0}\right)^{2}}\right),
\end{aligned}
$$

where $\omega_{p}$ is the plasma frequency, $\alpha$ is the density ratio of the ring electrons to the background positrons, $\gamma_{0}$ $=\sqrt{1+p_{0}^{2} / m_{0}^{2} c^{2}}$ is the Lorentz factor of a particle with momentum $p_{0}$ and rest mass $m_{0}$. Hereafter we normalize all the physical variables so that $\Omega_{0}=c=m_{0}=1$, and define $a$ $\equiv \omega_{p}^{2} / \Omega_{0}^{2}$.

A numerical solution of Eq. (4) with $\alpha=0.01$ and $a$ $=p_{0}=1$ is given in Fig. 1. The solid and dashed lines denote the real and the imaginary frequencies, respectively. Around $k_{\|}^{2} \approx 0$, the dispersion curves degenerate around the resonant frequency, $\omega \approx 1 / \gamma_{0}$. There are two unstable modes: the one with short wavelength $\left(k_{\|}^{2} / \omega^{2}>1\right)$ is the temperature anisotropy driven mode, and its maximum growth rate $\left(\delta_{l}\right)$ is obtained as

$$
\delta_{l}=\sqrt{\frac{\alpha a}{2}} \frac{p_{0}}{\gamma_{0}^{3 / 2}} .
$$

This mode is present also in a nonrelativistic plasma $\left(\gamma_{0}\right.$ $=1)$. The mode with long wavelength $\left(k_{\|}^{2} / \omega^{2}<1\right)$ has the maximum growth rate at $k_{\|} / \omega=0$,

$$
\delta_{l}=\sqrt{\frac{\alpha a}{2}} \frac{p_{0}}{\gamma_{0}^{3 / 2}} \sqrt{\frac{\gamma_{0}^{2}-1}{(1+2 a) \gamma_{0}^{2}-1}},
$$

and it is unstable only in the relativistic case, because the first term in the parentheses of the numerator of the sixth term $\left(\omega^{2}\right)$ vanishes in the nonrelativistic limit.

In obtaining Eqs. (5) and (6) we assumed $\omega \approx 1 / \gamma+\delta_{l}$ with an ordering, $O(a)=O\left(p_{0}\right)=\epsilon^{0}, \quad O(\alpha)=\epsilon^{2}$, and 
$O\left(\delta_{l}\right)=\epsilon^{1}$, where $\epsilon$ is a small parameter. We will discuss more in detail the analytical expressions for a variety of parameter regimes in $\mathrm{Sec}$. II C.

\section{B. Physical mechanism of the instability}

The ring system we defined in the previous subsection is at an equilibrium state if there is no (wave) electric field. However, when a small perturbation electric field is given to the system, the ring electrons are either accelerated or decelerated, depending on their relative phase to the phase of the electric field, and thus the ring electrons start to make differential Larmor rotation, caused by relativistic mass variation. This in turn leads to accumulation of the ring electron density at a certain phase angle, which equivalently acts as a feedback current to enhance the initially given electric field. We describe this mechanism in some detail below.

First we suppose that a small perturbation electric field is given to the system, $\mathbf{E}=E_{1}(\cos \eta, \sin \eta)$, where $\eta=\omega t$ is the phase of the electric field. This electric field will induce a perturbation in the ring distribution function, which we write as

$$
\begin{aligned}
& N(\phi, t)=\frac{n_{r}}{2 \pi}+N_{1}(t) \cos \left(\phi-\eta+\phi_{N}\right), \\
& P(\phi, t)=p_{0}+P_{1}(t) \cos \left(\phi-\eta+\phi_{P}\right),
\end{aligned}
$$

where $N(\phi, t)$ is the differential density of the ring distribution, $P(\phi, t)$ is the ring momentum radius, and $\phi$ is the phase angle (azimuthal angle) in the perpendicular momentum phase space (cf. the Appendix). The constants, $\phi_{N}$ and $\phi_{P}$, will be determined later. We include explicit time dependence in $N_{1}$ and $P_{1}$ (and also in $E_{1}$ ) for clarity of the discussion, but exactly the same argument can be made by letting these variables be constant, while allowing $\omega$ to be complex.

By using Eq. (A4) in the Appendix, to the first order, we have

$$
\begin{aligned}
& \dot{P}_{1} \cos \left(\phi-\eta+\phi_{P}\right)+\left(\omega+\frac{\Omega_{0}}{\gamma_{0}}\right) P_{1} \sin \left(\phi-\eta+\phi_{P}\right) \\
& =q E \cos (\phi-\eta),
\end{aligned}
$$

where the overdot represents a time derivative. The resonance condition between the wave and the ring particles is given by equating the coefficient of the second term to zero, $\omega+\Omega_{0} / \gamma_{0}=0$.

When the wave is far from the resonance, the above equation requires

$$
P_{1} \approx \frac{q E_{1}}{\omega+\Omega_{0} / \gamma_{0}} ; \quad \phi_{P} \approx \frac{\pi}{2} .
$$

This relation implies that the particles simply follow the rotating electric field $\pi / 2$ out of phase, so that there is no work acting on the particles.

When the wave satisfies the resonance condition, Eq. (9) reduces to

$$
\dot{P}_{1}=q E_{1} ; \quad \phi_{P}=0,
$$

i.e., the ring particles are constantly accelerated by the electric field, which the particles feel as dc. For each ring particle, the net momentum gain can be either positive or negative, as seen in Eq. (8). Hereafter we assume that the wave is in resonance with the ring particles, since this is the case leading to the instability. The background electrons and positrons, on the other hand, have different gyro-frequencies from the ring particles, and so their response to the electric field is given by Eq. (10).

As the ring particles gain momentum, their gyrofrequencies start to deviate from the zeroth order value,

$$
\frac{\Omega_{0}}{\gamma} \approx \frac{\Omega_{0}}{\gamma_{0}}\left(1-\frac{p_{0} P_{1}}{\gamma_{0}^{2}} \cos (\phi-\eta)\right) .
$$

The differential rotation results in an accumulation of the differential density: using the above equation and Eq. (A3) in the Appendix, we find

$$
\dot{N}_{1} \cos \left(\phi-\eta+\phi_{N}\right) \approx \frac{n_{r}}{2 \pi} \frac{\Omega_{0} p_{0} P_{1}}{\gamma_{0}^{3}} \sin (\phi-\eta) .
$$

Therefore, we have

$$
\dot{N}_{1} \approx \frac{n_{r}}{2 \pi} \frac{\Omega_{0} p_{0} P_{1}}{\gamma_{0}^{3}} ; \quad \phi_{N} \approx-\frac{\pi}{2}
$$

This density accumulation at a relative phase $\pi / 2$ from the electric field contributes to the generation of a perturbation current,

$$
\dot{J}_{1 \perp}=q \int_{0}^{2 \pi} \frac{\dot{N}_{1} p_{0}}{\gamma_{0}} \sin ^{2}(\phi-\eta) d(\phi-\eta)=\frac{q n_{r} \Omega_{0} p_{0}^{2} P_{1}}{2 \gamma_{0}^{4}}
$$

where we have only considered the current perpendicular to the electric field. Finally, from the perpendicular component of the Maxwell's equation $(\dot{\mathbf{E}}+\mathbf{J}=0)$, we have

$$
-\frac{\Omega_{0}}{\gamma_{0}} E_{1}+J_{1 \perp}=0
$$

where the resonance condition is used.

Thus from Eqs. (11), (15), (16), we have a set of evolution equations,

$$
\dot{P}_{1}=q E_{1} ; \quad \dot{E}_{1}=\frac{q n_{r} p_{0}^{2}}{2 \gamma_{0}^{3}} P_{1}
$$

which includes a purely growing eigenmode with the growth rate, $\left(q^{2} n_{r} p_{0}^{2} / 2 \gamma_{0}^{3}\right)^{1 / 2}=\left(\alpha a p_{0}^{2} / 2 \gamma_{0}^{3}\right)^{1 / 2}$. This is the main part of the linear growth rate Eq. (6) obtained in the previous section.

In order to be more precise, the di-electric response of the background as well as the ring species can be included in the discussion. Given the perturbation electric field $E_{1}$, the induced electric field influenced by the "bulk" motion of the plasma, is given as 


$$
\begin{aligned}
\frac{E_{\text {ind }}}{E_{1}} & =1-\frac{\omega_{p}^{2}}{\omega\left(\omega+\Omega_{0}\right)}-\frac{(1-\alpha) \omega_{p}^{2}}{\omega\left(\omega-\Omega_{0}\right)}-\frac{\alpha \omega_{p}^{2}}{\omega\left(\gamma_{0} \omega-\Omega_{0}\right)} \\
& =\frac{(1+2 a) \gamma_{0}^{2}-1}{\gamma_{0}^{2}-1},
\end{aligned}
$$

which essentially is the first five terms (except for the second term) in Eq. (4). Using $E_{\text {ind }}$ in place of $E_{1}$ in the second equation of Eq. (17), we recover Eq. (6).

From the argument above, it is clear that the relativistic ring instability is driven by the perturbation current Eq. (15) which is created by the accumulation of the ring electrons at a phase angle $\pi / 2$ off the phase of the wave electric field. Since the accumulation of the ring electrons is due to the differential Larmor rotation, Eq. (14), which is a direct consequence of the energy dependence of gyro-frequency, Eq. (12), the instability is intrinsically relativistic.

\section{Parametric survey of the dispersion relation}

The linear dispersion relation, Eq. (4), is numerically evaluated for the $k=0$ mode with various values of $a, p_{0}$, and $\alpha$. In Fig. 2, we show the real (solid lines) and the imaginary (dashed lines) frequencies plotted versus the initial ring momentum, $p_{0}$. As illustrated in panel (c), the dispersion curve can be divided into three different regimes, and the analytical approximation for the roots can be obtained for each regime as follows.

\section{Regime (i) $\left(p_{0}<p_{\mathrm{i}}\right)$}

When $p_{0}$ is small and of the order of $\epsilon$, then $\omega$ can be written as $\omega \approx 1 / \gamma+\omega_{1} \approx 1-p_{0}^{2} / 2+\omega_{1}+\cdots$, where $\omega_{1}$ is found to be of the order of $\epsilon^{3}$. From the lowest order of Eq. (4) we obtain

$$
\omega \approx 1-\frac{p_{0}^{2}}{2} \pm i \frac{\sqrt{\alpha} p_{0}^{2}}{2} .
$$

This approximation is shown as a dotted line in regime (i) in Fig. 2(c). The upper limit of this regime, $p_{\mathrm{i}}$, is determined later.

\section{Regime (ii) $\left(p_{\mathrm{i}}<p_{0}<p_{\mathrm{C}}\right)$}

When $p_{0} \gg 1$, it is appropriate to assume $p_{0}$ and $\gamma_{0}$ be of the order of $\epsilon^{-2}$, and $\omega$ of the order of $\epsilon^{2}$. Then the lowest order of Eq. (4) gives

$$
\omega=\frac{A+p_{0} B}{p_{0} A}\left(1 \pm \sqrt{1-\frac{A\left(A+3 p_{0} B\right)}{\left(A+p_{0} B\right)^{2}}}\right),
$$

where $A=2(1+2 a)$ and $B=\alpha a$. The marginal stability is given at

$$
p_{\mathrm{C}}=\frac{2(1+2 a)}{\alpha a} .
$$

Under the parameters used in Fig. 2(c), $p_{\mathrm{C}}=600$. When $p_{0}$ $<p_{\mathrm{C}}$, Eq. (20) is simplified as

$$
\omega \approx \frac{1}{p_{0}}+\frac{\alpha a}{2(1+2 a)} \pm i \sqrt{\frac{\alpha a}{2(1+2 a) p_{0}}} .
$$
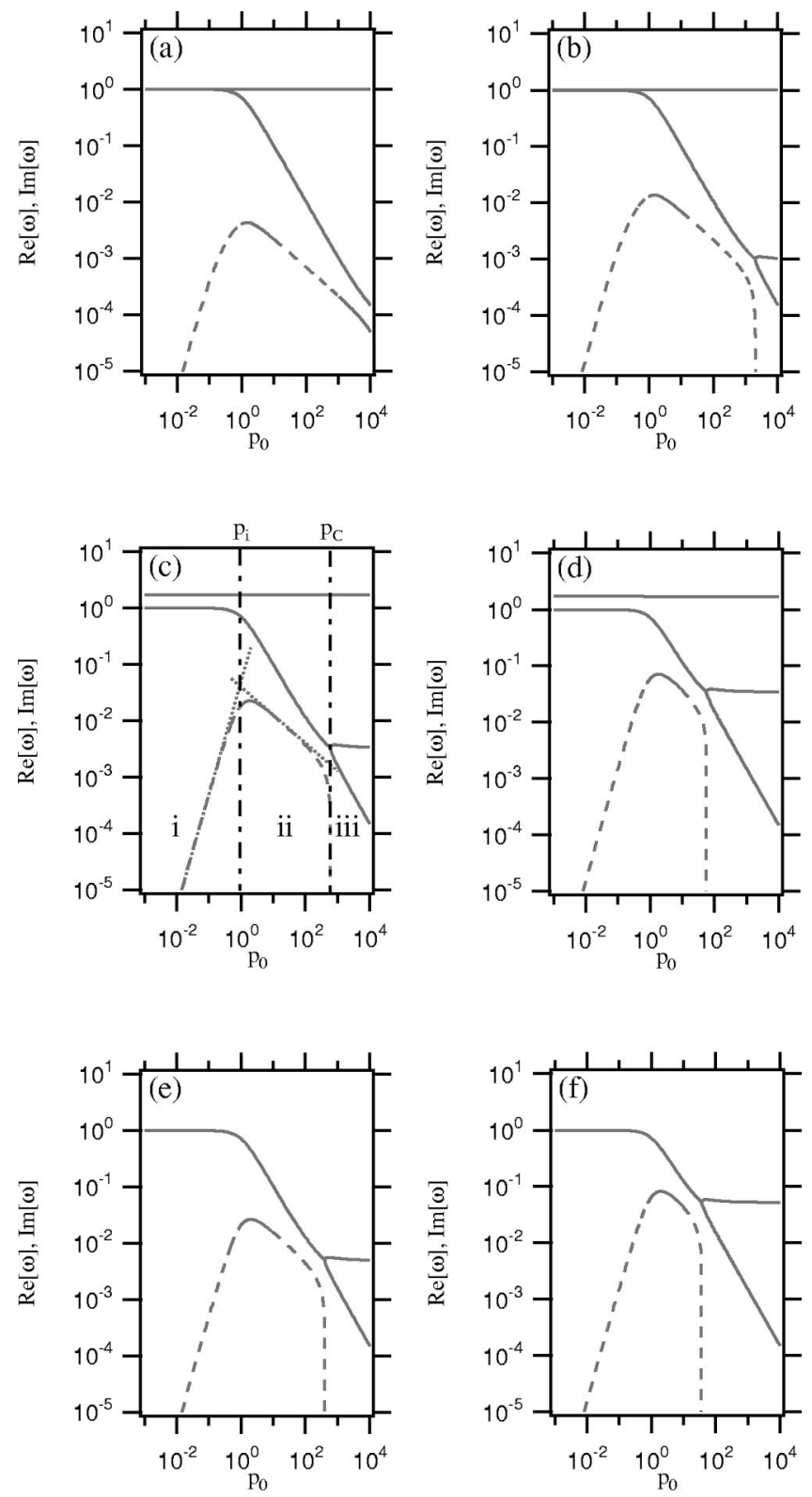

FIG. 2. Stability of the $k=0$ mode for various values of $a, p_{0}$, and $\alpha$. The solid and dashed lines represent the real and the imaginary parts of the frequency as a function of initial ring momentum $p_{0}$ for (a) $a=0.01, \alpha$ $=0.01$, (b) $a=0.01, \alpha=0.1$, (c) $a=1, \alpha=0.01$, (d) $a=01, \alpha=0.1$, (e) $a$ $=100, \alpha=0.01$, and (f) $a=100, \alpha=0.1$.

Comparing the imaginary parts of Eqs. (19) and (22), we find

$$
p_{\mathrm{i}}=\left(\frac{2 a}{\alpha(1+2 a)}\right)^{1 / 5} .
$$

This is shown as a dotted line in regime (ii) in Fig. 2(c).

\section{Regime (iii) $\left(p_{\mathrm{c}}<p_{0}\right)$}

When $p_{0}$ exceeds $p_{\mathrm{C}}$ defined above, the system becomes stable, and Eq. (20) gives two real roots,

$$
\omega_{1} \approx \frac{1}{2 p_{0}}+\frac{\alpha a}{1+2 a}, \quad \frac{3}{2 p_{0}} .
$$



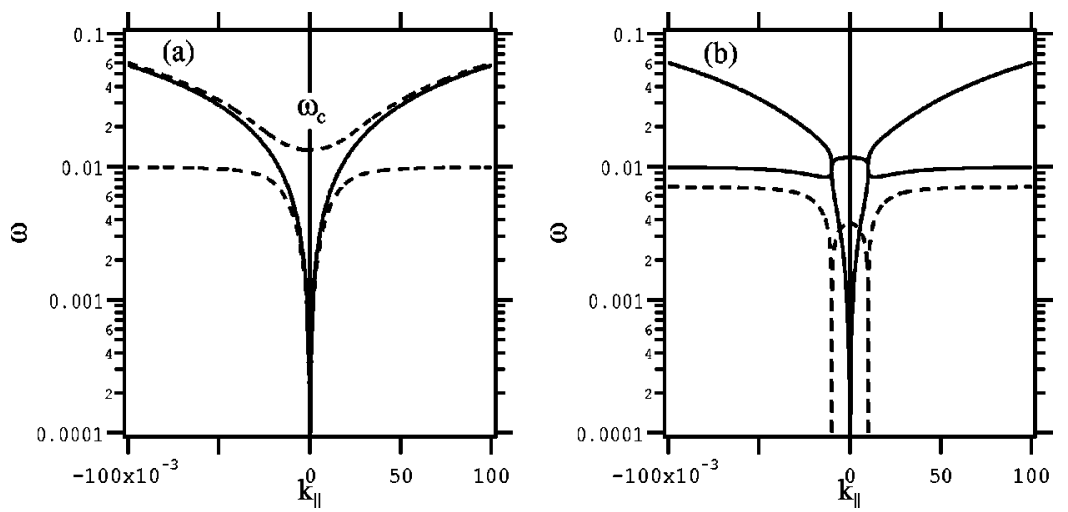

FIG. 3. Parallel dispersion relation of the electromagnetic waves [the solution of Eq. (4)] for $p_{0} \gg 1$. (a) The solid lines represent solutions of (4) in which both T1 and $\mathrm{T} 2$ are neglected, and dashed lines denote solutions with only T2 neglected but T1 included. (b) The solution of Eq. (4) for $\alpha=0.01, a=1, p_{0}=100$. The solid and dashed lines show the real and the imaginary frequencies.

The similar stabilization in an electron-proton plasma without background electrons is reported by Yoon and Davidson. ${ }^{20}$ The reason why the system is stabilized as the free energy is increased can be explained in terms of a loss of an energy channel from the ring electrons to the background plasma. This point can be understood by examining in detail the dispersion relation, Eq. (4), as follows.

The contribution of the ring electrons to the dispersion relation arises via the last two terms (terms in big parentheses) on the right-hand side (RHS) of Eq. (4), which we call $\mathrm{T} 1$ and T2 hereafter for brevity. In parameter regimes (ii) and (iii), the term T2 acts as the driving force of the instability, while T1 simply provides charge neutralization. Namely, without the term $\mathrm{T} 2$, the dispersion relation becomes similar to the one of a stable multi-component plasma with heavy (due to relativistic mass increase) plasma species. In Fig. 3(a), solid lines represent solutions of Eq. (4) in which both T1 and T2 are neglected, and dashed lines denote solutions with only T2 neglected but T1 included. Clearly, inclusion of the term T1 splits the dispersion curve into upper and lower branches, with the cut off frequency of the upper branch $\left(\omega_{\mathrm{c}}\right)$ at

$$
\omega_{\mathrm{c}} \approx \frac{1}{\gamma_{0}}+\frac{\alpha a p_{0}^{2}}{2 a+(1+2 a) p_{0}^{2}} .
$$

The instability takes place as the driving term T2 is also included [Fig. 3(b)], so that it can give away its energy at the resonance frequency, $\omega=1 / \gamma_{0}$. Since $\omega_{\mathrm{c}}$ is close to $\left(1 / \gamma_{0}\right)$ only when $p_{0} \ll p_{\mathrm{C}}$, the system is destabilized as $p_{0}$ exceeds $p_{C}$.

\section{DISPERSION RELATION FOR NONGYROTROPIC RING DISTRIBUTION}

In this section we extend our analysis to a nongyrotropic distribution function, which are often detected in space plasmas. A number of peculiar and interesting features of the instabilities driven by the nongyrotropic distribution functions have been discussed extensively by many authors (e.g., Sudan, ${ }^{21}$ Brinca et al., ${ }^{22}$ Cao et al. ${ }^{23}$ and Freund et al. $\left.{ }^{24}\right)$. Among them is the presence of the coupling between the right- and the left-hand polarized electromagnetic waves found in a gyrotropic plasma and also the electrostatic waves even for parallel propagation.
We start from the Vlasov equation in the cylindrical coordinate,

$$
\left(\frac{\partial}{\partial t}-\frac{\Omega_{0}}{\gamma} \frac{\partial}{\partial \phi}\right) f_{j}=-q \mathbf{E} \cdot \frac{\partial f_{j}}{\partial \mathbf{p}},
$$

where $\phi$ is the gyrophase defined by $\phi=\tan ^{-1}\left(p_{y} / p_{x}\right)$, and other physical quantities are

$$
\begin{aligned}
& f_{j}=f_{0 j}+\delta f_{j}, \\
& \mathbf{E}=\delta \mathbf{E}, \\
& \mathbf{B}=\mathbf{B}_{0} .
\end{aligned}
$$

The presence of the nongyrotropy $(\partial / \partial \phi \neq 0)$ in the ring electrons implies the nonstationarity of $f_{0}$ : it should have the form

$$
\begin{aligned}
& f_{0}=\sum_{n=-\infty}^{\infty} G_{n}\left(p_{\|}, p_{\perp}\right) \mathrm{e}^{-i n(\phi+\Omega t)}, \\
& G_{n}=\frac{1}{2 \pi} \int f_{0} \mathrm{e}^{i n(\phi+\Omega t)} \mathrm{d}(\phi+\Omega t),
\end{aligned}
$$

where $\Omega=\Omega_{0} / \gamma$, and we dropped the subscript $r$ for simplicity. The zeroth order distribution function, Eq. (27), is a solution of Eq. (25).

Linearizing and Fourier transforming Eq. (25),

$$
\begin{gathered}
\left(-i \omega-\frac{\Omega_{0}}{\gamma} \frac{\partial}{\partial \phi}\right) \delta f(\omega) \\
=-q \sum_{n} \delta \mathbf{E}(\omega-n \Omega) \cdot \frac{\partial}{\partial \mathbf{p}} G_{n} \mathrm{e}^{-i n \phi} \\
=-q \sum_{n}\left[\left(\frac{\partial G_{n}}{\partial p_{\perp}}-\frac{n}{p_{\perp}} G_{n}\right) E_{+} \mathrm{e}^{-i(n+1) \phi}\right. \\
\left.\quad+\left(\frac{\partial G_{n}}{\partial p_{\perp}}+\frac{n}{p_{\perp}} G_{n}\right) E_{-} \mathrm{e}^{-i(n-1) \phi}\right],
\end{gathered}
$$

where $E_{ \pm}=\left(E_{x} \pm i E_{y}\right) / 2$. Putting $\delta f(\omega)=\Sigma_{n} f_{n}(\omega)$ $\exp (-i n \phi)$ and comparing the coefficients of identical harmonics $(\exp (-i n \phi))$, we have 


$$
\begin{aligned}
f_{n}(\omega)= & -i \frac{q}{\omega-n \Omega}\left[\left(\frac{\partial G_{n-1}}{\partial p_{\perp}}-\frac{n-1}{p_{\perp}} G_{n-1}\right)\right. \\
& \times E_{+}(\omega-(n-1) \Omega)+\left(\frac{\partial G_{n+1}}{\partial p_{\perp}}+\frac{n+1}{p_{\perp}} G_{n+1}\right) \\
& \left.\times E_{-}(\omega-(n+1) \Omega)\right] .
\end{aligned}
$$

Ampere's law together with Eq. (30) leads to

$$
\begin{aligned}
\omega E_{ \pm}(\omega)= & -4 \pi q^{2} \sum_{j} \int \mathrm{d} p_{\|} \int \mathrm{d} p_{\perp} \frac{p_{\perp}^{2}}{2 \gamma}\left[\frac{E_{ \pm}(\omega)}{\omega \mp \Omega} \frac{\partial G_{0}}{\partial p_{\perp}}\right. \\
& \left.+\frac{E_{\mp}(\omega \mp 2 \Omega)}{\omega \mp \Omega}\left(\frac{\partial G_{ \pm 2}}{\partial p_{\perp}}+\frac{2}{p_{\perp}} G_{ \pm 2}\right)\right] .
\end{aligned}
$$

The second term on the RHS of Eq. (31) comes from the nongyrotropy of the ring electrons. If $f_{0}$ is written as $f_{0}$ $=F\left(p_{\|}, p_{\perp}\right) \Phi(\phi+\Omega t)$, where $\Phi(\phi+\Omega t)=(1 / 2 \pi) \Sigma_{n} \Phi_{n}$ $\exp (-i n(\phi+\Omega t))$ and $\Phi_{n}=\int \Phi \exp (i n(\phi+\Omega t)) \mathrm{d}(\phi+\Omega t)$, then $G_{n}=F \Phi_{n} / 2 \pi$. As pointed out by Brinca et al. ${ }^{22}$ the higher order $\left(G_{n},|n| \geqslant 3\right)$ harmonics do not contribute to the linear dispersion relation. This is easily confirmed by using the expression of $\Phi$,

$\Phi(\phi+\Omega t)=\left\{\begin{array}{l}\frac{1}{m} \sum_{l=0}^{m-1} \delta\left(\phi+\Omega t+\frac{2 \pi l}{m}\right) \quad(m \geqslant 2), \\ \frac{1}{2 \pi} \quad(m=0) .\end{array}\right.$

Here the case $m=1$ is excluded, since this choice of $m$ violates the initial assumption of a null zeroth order electric field. The terms $G_{ \pm 2}$ are finite only when $m=2$, so that the perturbation current for $m \geqslant 3$ becomes identical to that of the gyrotropic case $(m=0)$.

While paying attention to the momentum dependence to the arguments of $E_{ \pm}$, the term including $\partial G_{ \pm 2} / \partial p_{\perp}$ in Eq. (31) becomes

$$
\begin{aligned}
-\int \mathrm{d} p_{\perp}\left\{\left[\frac{\partial}{\partial p_{\perp}}\left(\frac{p_{\perp}^{2}}{2\left(\gamma \omega \mp \Omega_{0}\right)}\right)\right] E_{\mp}(\omega \mp 2 \Omega)\right. \\
\left.+\left(\frac{p_{\perp}^{2}}{2\left(\gamma \omega \mp \Omega_{0}\right)}\right)\left[\frac{\partial(\omega \mp 2 \Omega)}{\partial p_{\perp}} \frac{\partial E_{\mp}(\omega \mp 2 \Omega)}{\partial(\omega \mp 2 \Omega)}\right]\right\} G_{ \pm 2} .
\end{aligned}
$$

If we assume $F=\left(1 / p_{\perp}\right) \delta\left(p_{\|}\right) \delta\left(p_{\perp}-p_{0}\right)$, then the second term in Eq. (33) is proportional to $\partial E_{\mp}(\omega \mp 2 \Omega) / \partial(\omega$ $\mp 2 \Omega)\left.\right|_{\omega=\omega_{0}}$, where $\omega_{0}=\omega\left(p_{\perp}=p_{0}\right)$, and is equal to zero, and the arguments of $E_{ \pm}$become independent of the momentum. Then, we arrive at two equations relating $E_{ \pm}$:

$$
\begin{aligned}
{[\omega} & \left.-\sum_{j}\left(\frac{\omega_{p}^{2}}{\gamma \omega \mp \Omega_{0}}-\frac{\omega_{p}^{2} p_{0}^{2} \omega}{2 \gamma\left(\gamma \omega \mp \Omega_{0}\right)^{2}}\right) G_{0}\right] E_{ \pm}(\omega) \\
+ & {\left[\sum_{j} \frac{\omega_{p}^{2} p_{0}^{2} \omega}{2 \gamma\left(\gamma \omega \mp \Omega_{0}\right)^{2}} G_{ \pm 2}\right] E_{\mp}(\omega \mp 2 \Omega)=0 . }
\end{aligned}
$$

The final dispersion relation is given by requiring the above relations to have nontrivial solutions (zero-determinant).

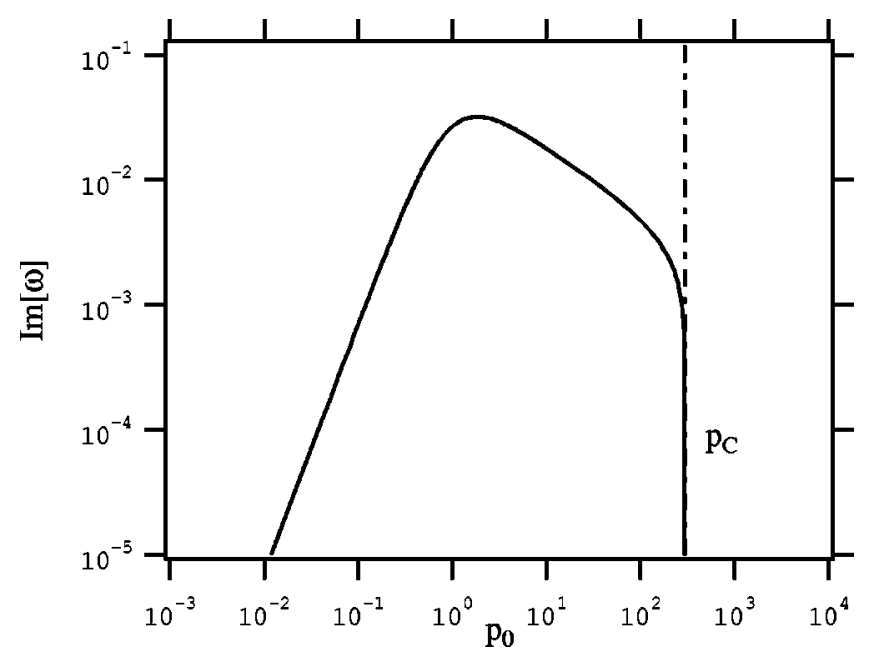

FIG. 4. The growth rate of the $k=0$ mode in the nongyrotropic plasma with $m=2$ for $a=1$ and $\alpha=0.01$ as a function of $p_{0}$.

The expression of the linear growth rate at the same ordering of parameters in obtaining Eq. (6) is derived as

$$
\delta_{l}=\sqrt{\alpha a} \frac{p_{0}}{\gamma_{0}^{3 / 2}} \sqrt{\frac{\gamma_{0}^{2}-1}{(1+2 a) \gamma_{0}^{2}-1}} .
$$

This is slightly larger than the gyrotropic case, because the current induced by the perturbation is enhanced when $m$ $=2$. Figure 4 shows the numerical solution of the growth rate for $a=1$ and $\alpha=0.01$. Dependence to $p_{0}$ similar to the gyrotropic case is observed. However, $p_{\mathrm{C}}$ is slightly less than the gyrotropic plasma.

\section{NUMERICAL SIMULATIONS}

In the present study we drop spatial dependence of all the physical variables (i.e., we consider a zero-dimensional system) so that we can focus on time evolution of the instability driven solely by the relativistic mass variation; this way, we can discuss nonlinear evolution and saturation of the system with extremely high accuracy. The relativistic electromagnetic full particle code is used to follow nonlinear time evolution of the instability, in which we simultaneously solve the Ampere's law with $\boldsymbol{\nabla}=0$ and the equations of motion of particles. We confirmed that this instability is observed for a wide range of parameters as the linear theory predicts. The simulation parameters used for each run are summarized in Table I.

\section{A. Run $1\left(p_{0}<p_{C}\right)$ for gyrotropic ring distribution}

Here we show time evolution of the system for the gyrotropic ring distribution with $p_{0}=5\left(<p_{C}\right)$ (run 1). Figure 5 shows the snapshots of the distribution of ring electrons, in the momentum phase space $\left(p_{x}-p_{y}\right)$ (left panels), and the phase space of $\Delta p$ and $\varphi_{r}$ (right panels), where $\Delta p$ is the deviation of the ring momentum from its initial value, and the phase difference $\varphi_{r}$ is the difference between the phase of each particle to the phase of the electric field.

At $t=300$, the distribution is almost the same as initial conditions. At later times $(t=600,800)$, the ring is gradually 
TABLE I. Parameters for simulation runs.

\begin{tabular}{|c|c|c|c|c|c|c|c|c|}
\hline \multicolumn{9}{|l|}{ Fixed parameters } \\
\hline \multirow[t]{3}{*}{ Initial particle numbers } & \\
\hline & \multicolumn{8}{|c|}{$n_{p}=10000$} \\
\hline & \multicolumn{8}{|c|}{$n_{r}=10000$} \\
\hline \multirow{5}{*}{$\begin{array}{l}\text { Density of ring } \\
\text { Initial thermal spread } \\
a\left(=\omega_{p}^{2} / \Omega_{0}^{2}\right)\end{array}$} & \multirow{3}{*}{\multicolumn{8}{|c|}{$\begin{array}{c}n_{r} / n_{p}=0.01 \\
p_{\text {th }} / p_{0}=0.001 \\
a=1.0\end{array}$}} \\
\hline & & & & & & & & \\
\hline & & & & & & & & \\
\hline & \multicolumn{8}{|c|}{ Simulations for gyrotropic ring distributions $(m=0)$} \\
\hline & run 1 & run 2 & run 3 & run 4 & run 5 & run 6 & run 7 & run 8 \\
\hline Time step: $\Omega_{0} \Delta t$ & 0.01 & 0.01 & 0.01 & 0.1 & 0.1 & 0.1 & 0.1 & 0.1 \\
\hline \multirow[t]{2}{*}{ Initial ring momentum: $p_{0} / m_{0} c$} & 5.0 & 10.0 & 50.0 & 100.0 & 150.0 & 200.0 & 250.0 & 300.0 \\
\hline & run 9 & run 10 & run 11 & run 12 & run 13 & run 14 & run 15 & run 16 \\
\hline$\Omega_{0} \Delta t$ & 0.1 & 0.1 & 0.1 & 0.1 & 0.1 & 0.1 & 0.1 & 0.1 \\
\hline \multirow[t]{2}{*}{$p_{0} / m_{0} c$} & 350.0 & 400.0 & 450.0 & 500.0 & 550.0 & 600.0 & 601.855 & 606.0 \\
\hline & run 17 & run 18 & run 19 & run 20 & run 21 & & & \\
\hline$\Omega_{0} \Delta t$ & 0.1 & 0.1 & 0.1 & 0.1 & 0.1 & & & \\
\hline \multirow[t]{3}{*}{$p_{0} / m_{0} c$} & 620.0 & 650.0 & 700.0 & 750.0 & 800.0 & & & \\
\hline & \multicolumn{8}{|c|}{ Simulations for nongyrotropic ring distributions $(m \geqslant 2)$} \\
\hline & \multicolumn{2}{|c|}{ run 22} & \multicolumn{2}{|c|}{ run 23} & & 24 & \multicolumn{2}{|c|}{ run 25} \\
\hline$m$ & \multicolumn{2}{|c|}{2} & \multicolumn{2}{|c|}{2} & & 3 & \multicolumn{2}{|c|}{3} \\
\hline$\Omega_{0} \Delta t$ & \multicolumn{2}{|c|}{0.01} & \multicolumn{2}{|c|}{0.1} & & 01 & \multicolumn{2}{|c|}{0.1} \\
\hline$p_{0} / m_{0} c$ & \multicolumn{2}{|c|}{5.0} & \multicolumn{2}{|c|}{301.0} & & 0 & \multicolumn{2}{|c|}{601.0} \\
\hline
\end{tabular}

broken and steepens around a stationary point at $\varphi_{r}=\pi / 2$. This steepening of the ring in the momentum space is characteristic of the relativistic effect, i.e., the larger the momentum is, the less the cyclotron frequency becomes. Later at $t$ $=6,500$, it is seen that the majority of particles bunch around the stationary point, although the rest of the particles also almost fill the separatrix, which is recognizable in the phase space of $\Delta p$ and $\varphi_{r}$. At this stage it is expected that the instability has already terminated due to the particle trapping.

In Fig. 6, time history of the electric field energy for the same run is shown. It is confirmed that the time development of the system is divided into two stages: the linear growth and subsequent nonlinear saturation. The oscillation in the nonlinear stage corresponds to the trapping oscillation of the ring electrons.

Saturation level. The saturation mechanism of this instability is the phase bunching of the ring electrons. The saturation level can be estimated by modeling the final state of the instability, which is obtained from simulations. We illustrate our model in Fig. 7. After sufficient time, it is expected that the electric field amplitude becomes constant. In addition, we assume that the phase relations between the wave and each plasma component becomes as follows: (1) all the ring electrons concentrate at the stationary point $\left(\varphi_{r}\right.$ $=\pi / 2$ ), making a single cluster, (2) the background electrons and positrons shift in the momentum phase space $\left(\varphi_{e, p}=-\pi / 2\right)$ so that the total electric force is canceled. Therefore, the equations we consider are the equations of motion for the three plasma components, Ampere's law, and the conservation of total energy,

$$
\begin{aligned}
& \dot{\mathbf{p}}_{s}=q_{s} \mathbf{E}+\frac{\mathbf{p}_{s}}{\gamma_{s}} \times \Omega_{0 s} \hat{\mathbf{z}}, \\
& 0=\frac{1}{n_{0}} \mathbf{j}+\dot{\mathbf{E}}, \\
& \mathbf{W}_{\text {kinetic }}(0)=\mathbf{W}_{\text {field }}\left(t_{\text {sat }}\right)+\mathbf{W}_{\text {kinetic }}\left(t_{\text {sat }}\right) .
\end{aligned}
$$

The saturation level is determined by solving these equations simultaneously. Here the subscript $s$ represents the plasma species $(e, p$; background electrons and positrons, $r$; ring electrons), $q_{e, r}=\Omega_{0 e, r}=-1, q_{p}=\Omega_{0 p}=1$, and the dot represents the time derivative. These equations can be rewritten as

$$
\begin{aligned}
& \dot{p}_{s}=q_{s} E_{\mathrm{sat}} \cos \varphi_{s}, \\
& \dot{\varphi}_{s}=-\frac{q_{s}}{p_{s}} E_{\mathrm{sat}} \sin \varphi_{s}-\frac{q_{s}}{\gamma_{s}}-\omega, \\
& \sum_{s} \alpha_{s} q_{s} \frac{p_{s}}{\gamma_{s}} \cos \varphi_{s}=0, \\
& \omega E_{\mathrm{sat}}+\sum_{s} \alpha_{s} q_{s} \frac{p_{s}}{\gamma_{s}} \sin \varphi_{s}=0, \\
& \frac{E_{\mathrm{sat}}^{2}}{2}+\sum_{s} \alpha_{s} \gamma_{s}=\sum_{s} \alpha_{s} \gamma_{\mathrm{s} 0},
\end{aligned}
$$



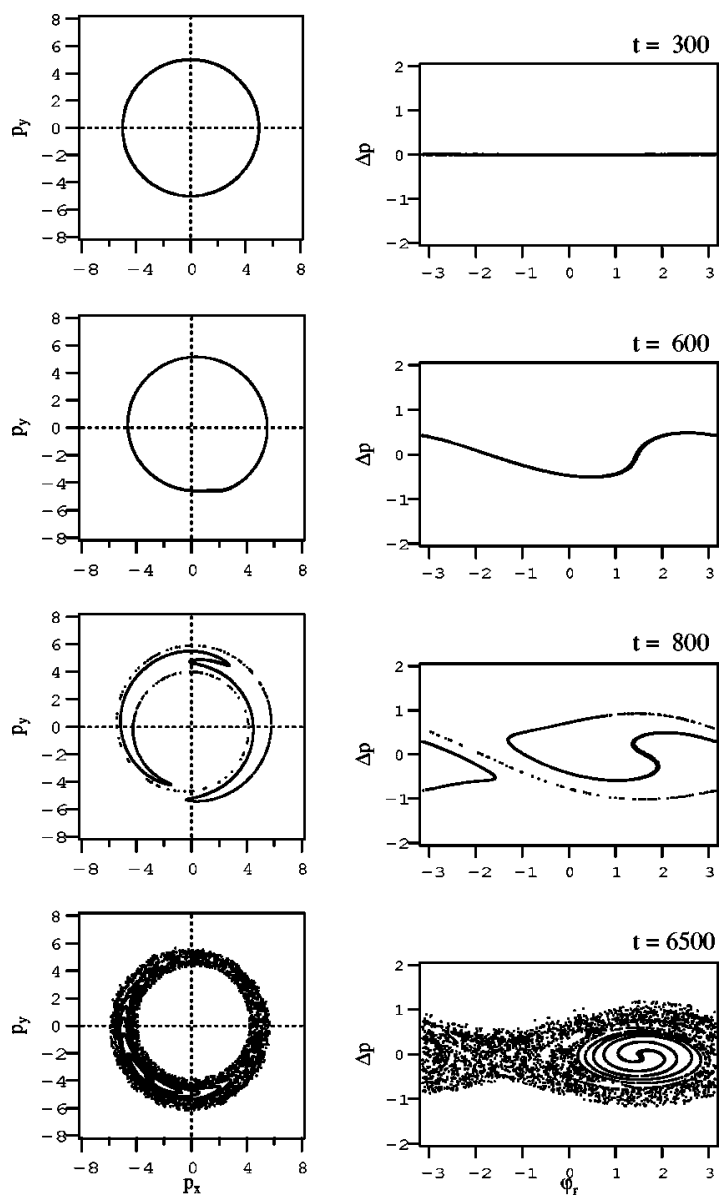

FIG. 5. Time evolution of the ring distribution for run 1 . The panels on the left side represent the distributions in the perpendicular momentum phase space $\left(p_{\mathrm{x}}-p_{\mathrm{y}}\right)$. In the panels on the right side, the horizontal axes indicate the relative phase $\left(\varphi_{r}\right)$ of each particle to the wave electric field, and the vertical axes are the deviation of momentum from its initial value $(\Delta p)$.

where $\varphi_{s}$ is the phase difference between the phase of each particle species to the phase of the electric field, and $\alpha_{s}$ is the density ratio of species $s$ to total plasma.

When $p_{0}$ and $a$ are of the order of $\epsilon^{0}$, we find the saturation level of the electric field to be given by

$$
E_{\text {sat }}=\frac{\alpha_{0}\left(\gamma_{0}^{2}-1\right)}{(1+2 a) \gamma_{0}^{2}-1} \text {. }
$$

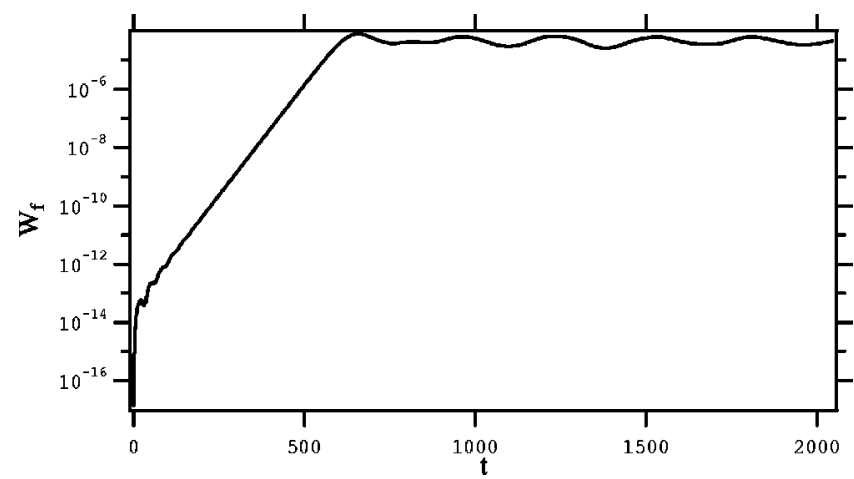

FIG. 6. The time history of the electric field energy for run 1.

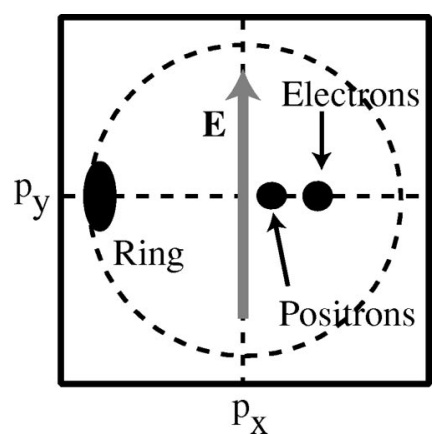

FIG. 7. The model of the saturation state (single cluster) on the frame rotating with the wave electric field.

Figure 8 compares the saturation level of the electric field energy obtained by the model above (solid line) and the simulation results (dots), as a function of the initial ring momentum, $p_{0}$. As $p_{0}$ is increased the wave saturation level increases also. However, when $p_{0}$ is further increased beyond the critical momentum $p_{C}$ defined in Eq. (21), the saturation level drops significantly, although it is still considerably larger than the noise level, which is typically around $10^{-6}$. In the next section, we analyze the results of the another simulation run (run 15) in which $p_{0}$ is just above $p_{C}$.

\section{B. Run $15\left(p_{0}>p_{\mathrm{C}}\right)$ with gyrotropic ring distribution}

When $p_{0}>p_{\mathrm{C}}$, the linear theory predicts the system to be stable, but the numerical simulation shows that the system is driven unstable after a sufficiently long time. Figure 9 shows the time history of the wave electric field energy for run $15\left[p_{0}=601.855\left(>p_{\mathrm{C}}\right)\right]$, which can be divided into three (instead of two as in run 1) different stages. Characteristic about this run is the first stage $(0<t<100,000)$, in which the wave energy lingers at a relatively low level compared with the final wave energy in stage III, although even at this time the wave energy is much higher than the noise level. Then, the linear instability is suddenly triggered around $t=100,000$, indicating the beginning of the second stage. The evolution is then followed by the saturation $(t$ $>110,000)$.

The distribution of ring electrons in the stage $\mathrm{I}$ is shown in the phase space of $\Delta p$ and $\varphi_{r}$ in the upper panel of Fig.

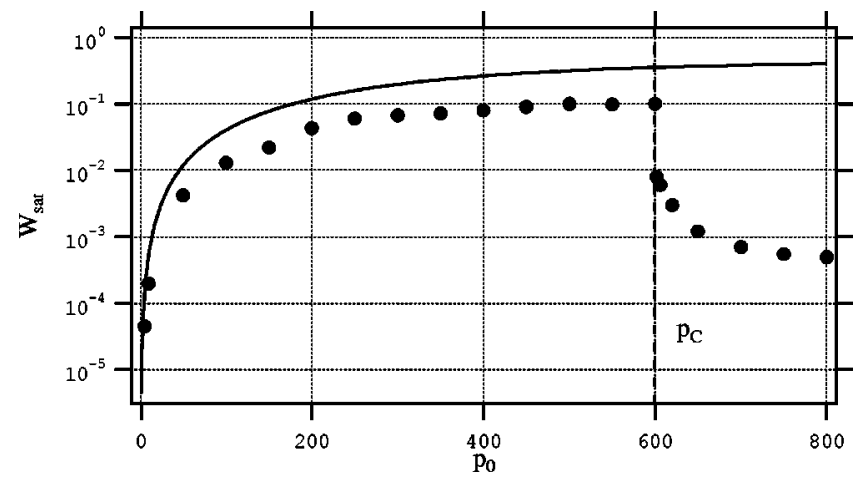

FIG. 8. The saturation level of the electric field energy as a function of initial ring momentum. The solid line is obtained by solving Eqs. (36), (37), and (38), the dots indicate the results of numerical simulations. 


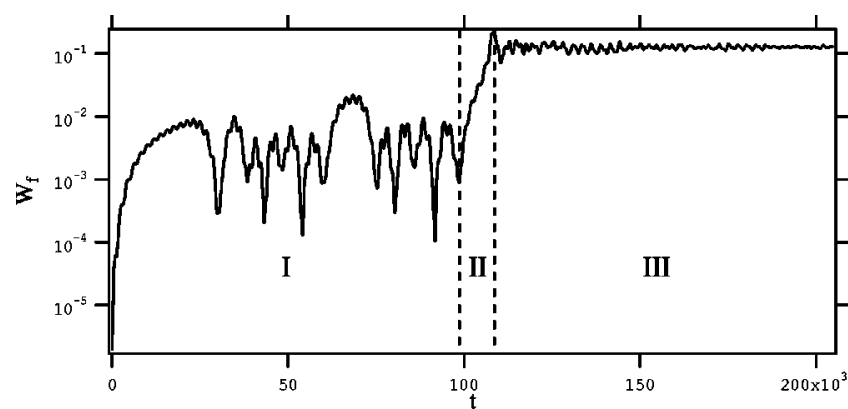

FIG. 9. The time history of the electric field energy for run 15 .

10. Clearly, two stationary points separated by $\sim \pi$ in $\varphi_{r}$ have appeared in the phase space, and many ring electrons have started to bunch around them, making a double-cluster structure. This structure is not time stationary, but it oscillates back and forth in the direction of $\Delta p$, and is quite stable as already shown in Fig. 9. In Fig. 8, the "saturation" levels plotted for $p_{0}>p_{C}$ correspond to the wave energy in stage I. After a long time, this metastable state eventually evolves to the single cluster state (the lower panel of Fig. 10), accompanied with much larger wave energy than that with the double-cluster state.

Quasi-saturation mechanism. As we discussed before, according to the linear theory, when $p_{0}$ is increased and exceeds $p_{C}$, the degeneracy of wave modes around $\omega \approx 1 / \gamma_{0}$ is resolved, and two wave modes appear around $k=0$. The frequencies of these waves are close to the ring cyclotron fre-
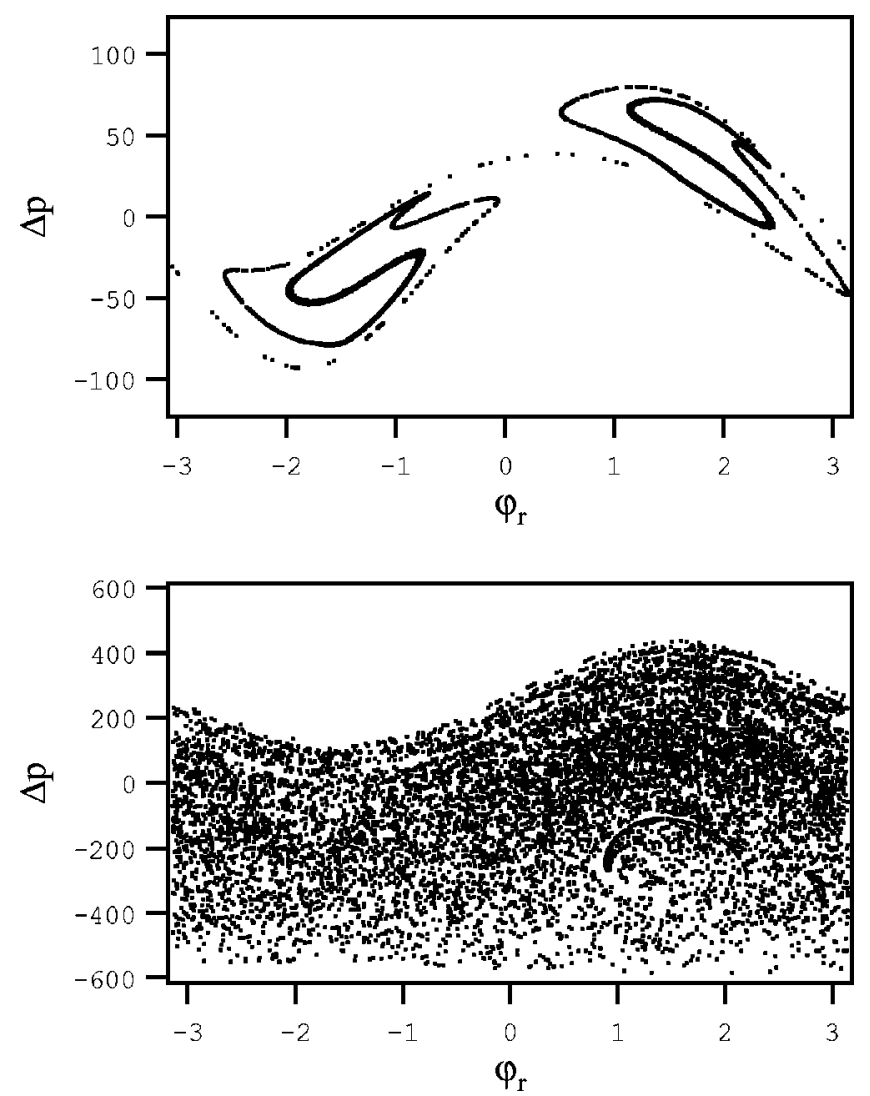

FIG. 10. Ring distributions in the $\Delta p-\varphi_{r}$ phase space at stages I (upper panel) and III (lower panel) for run 15.

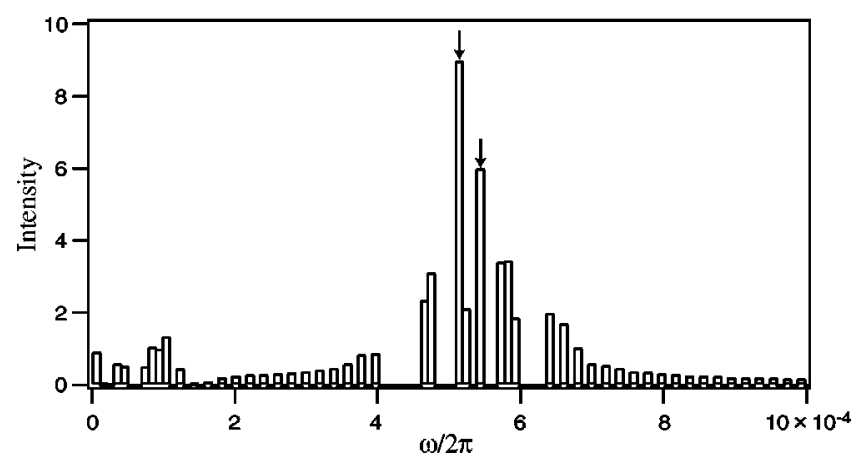

FIG. 11. The frequency spectrum of the wave electric field in stage I for run 15 .

quency $1 / \gamma_{0}$, but electrons resonate with neither of these waves, since the ring electrons are located outside the separatrices in the phase space. Figure 11 shows the frequency spectrum of the wave electric field for $0<t<51,000$, which is within stage I. Although the wave energy is distributed in a rather wide range of frequencies, caused presumably by spreading of the ring electrons, wave-wave interactions, and the finite sampling time scale of the data, two peaks (indicated by arrows), corresponding to the two waves mentioned above, are eminent.

The presence of two waves naturally produces the beat wave. The beat period estimated from the two peaks in Fig. 11 is consistent with the oscillation period of the field energy in the early period of stage I in Fig. 9. In the dynamical system for a particle interacting with two waves, for instance, it is well known that there appears the secondary resonance outside of the separatrix of main resonance. The double-cluster state, here, corresponds to it.

\section{Numerical simulations for nongyrotropic ring distribution}

Here the time evolution of nongyrotropic ring instability is investigated. The linear theory shows that the ring distribution function with $m=2$ [where $m$ is the azimuthal nongyrotropy number defined in Eq. (32)] makes the growth rate slightly larger, and $p_{C}$ slightly less than the gyrotropic case, while the linear dispersion properties remain unchanged when the distribution function with $m \geqslant 3$. In the following

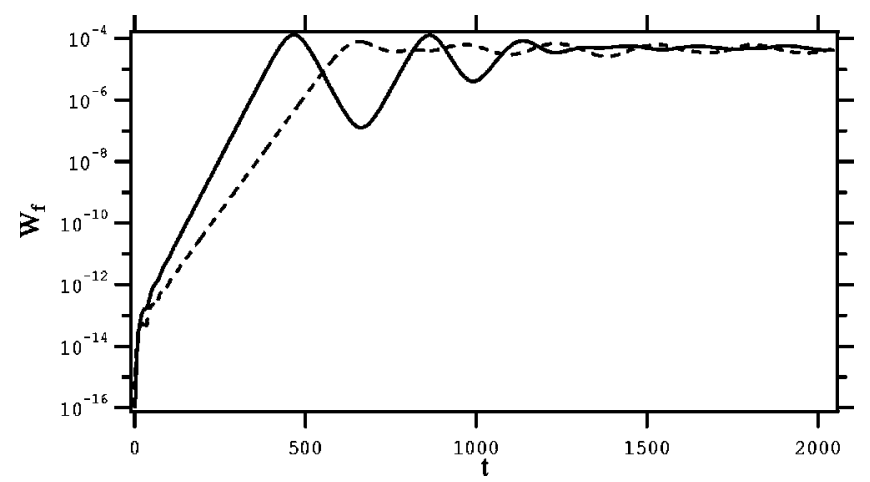

FIG. 12. The time history of the electric field energy for run 22. The dashed line, which corresponds to the gyrotropic case, is added for comparison. 
we show numerical simulation results indicating the differences between the gyrotropic and nongyrotropic ring distributions.

\section{Runs 22 and $23(m=2)$}

In Fig. 12, time history of the wave energy for run 22 [nongyrotropic with $m=2$ and $p_{0}=5\left(<p_{C}\right)$, solid line] is compared with the gyrotropic run with the same parameters (dashed line). As the linear theory predicts, when the nongyrotropic distribution is used, the linear growth rate is enhanced, and also the amplitude and time period of nonlinear oscillations are larger compared with the gyrotropic run.

Figure 13 represents time evolution of the ring electrons, presented in the same format as Fig. 5. $\mathrm{C} 1$ and $\mathrm{C} 2$ denote the two clusters. In the linear stage $(t \leqslant 400)$, the electrons at $\mathrm{C} 1$ provide energy to the wave $(\mathbf{p} \cdot \mathbf{E}<0)$, while those at $\mathrm{C} 2$ absorb energy from the wave $(\mathbf{p} \cdot \mathbf{E}>0)$. The wave resonates more efficiently with the electrons at $\mathrm{C} 1$ than those at $\mathrm{C} 2$ because the deviation of the phase of $\mathrm{C} 2$ from its initial value is greater than that of $\mathrm{C} 1$. After a long time at $t$ $=1,400$, the instability is already saturated, represented by electrons at $\mathrm{C} 1$ and $\mathrm{C} 2$ both being trapped by the finite amplitude wave.

Figure 12 compares the time evolution of the wave energy for the nongyrotropic (solid line) and the gyrotropic (dashed line) runs made by using the same parameters. For the nongyrotropic run, since the electrons were initially lo-
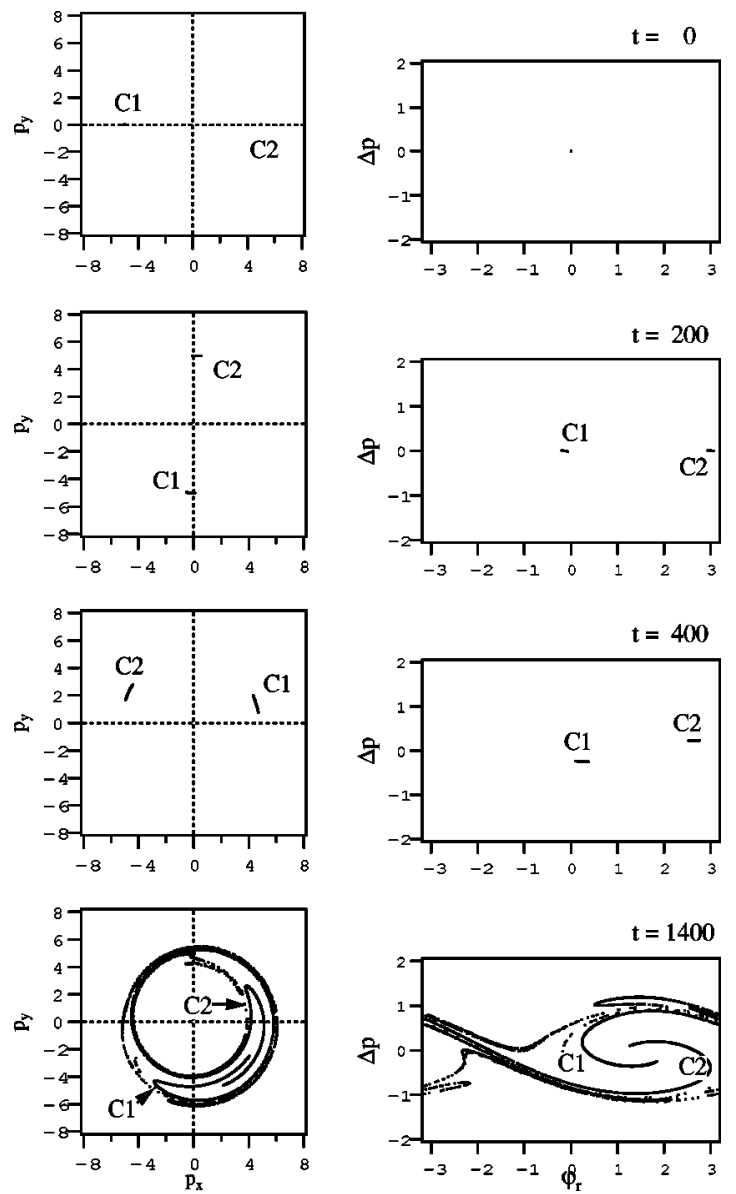

FIG. 13. The time evolution of the ring distribution for run 22 .

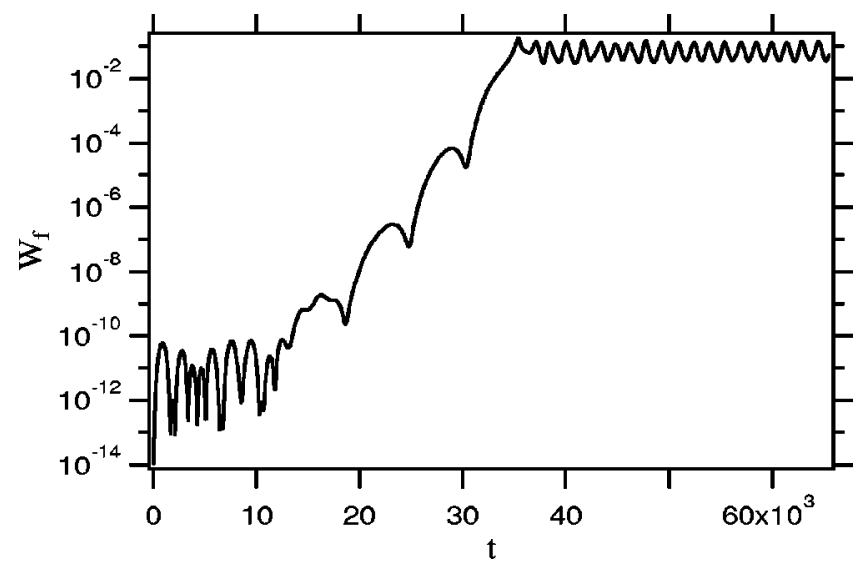

FIG. 14. The time history of the electric field energy for run 23 .

cated near the trapping separatrix (as seen in Fig. 13), trapping oscillation has a longer time period and larger amplitude compared with the gyrotropic run. On the other hand, the eventual saturation level is almost equal for both runs, due to the mixing within the separatrix.

The time history of wave energy for run $23\left[p_{0}=301\right.$ $\left.\left(>p_{\mathrm{C}}\right)\right]$ is shown in Fig. 14. The wave energy gradually grows in this case too, despite of that the system is linearly stable.

\section{Runs 24 and $25(m=3)$}

When the ring electrons have a nongyrotropy with $m$ $=3$, the characteristics of the instability becomes the same as the gyrotropic case $(m=0)$. Figure 15 compares time histories of the wave energy obtained from runs with $m=3$ (solid line) and $m=0$ (dashed line). In both cases, $p_{0}=5\left(<p_{C}\right)$. Both at the linear and nonlinear stages, no apparent differences can be recognized between the two runs, except for a little difference in the fluctuation amplitude of the wave energy after the saturation.

Figure 16 represents snapshots of the ring electrons drawn in the same format as Fig. 5. The time development of the clusters are quite different depending on their initial positions in the phase space. One of the clusters, $\mathrm{C} 2$, was initially near the saddle point at $\varphi=-\pi / 2$. At $t=1,400$, it was strongly elongated along the separatrix. On the other hand, $\mathrm{C} 1$ and $\mathrm{C} 3$ only followed the equi-energy contours without

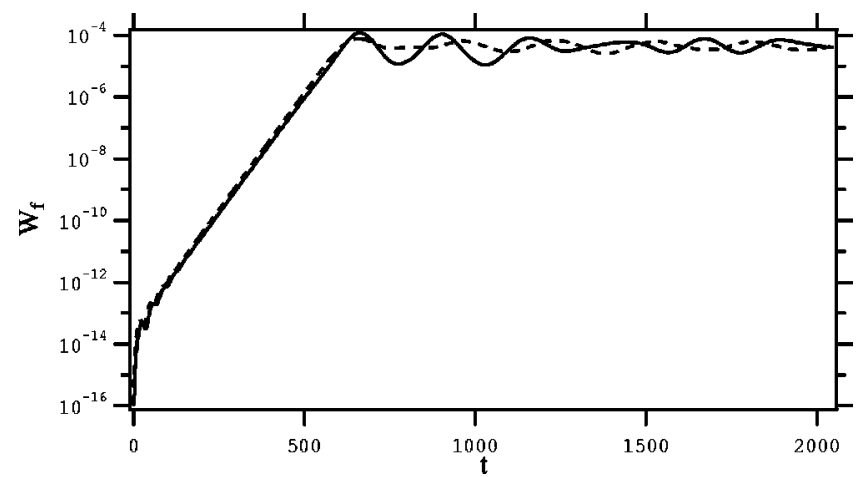

FIG. 15. The time history of the electric field energy for run 24 . The dashed line, which corresponds to the gyrotropic case, is added for comparison. 

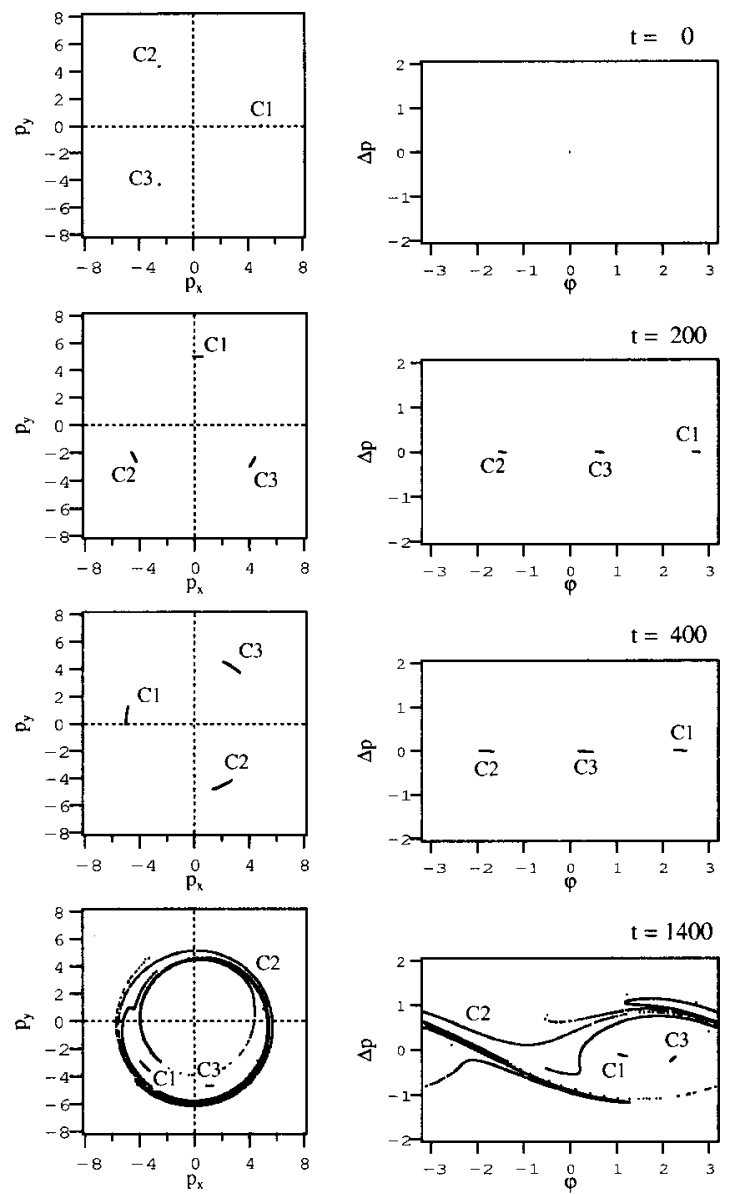

FIG. 16. The time evolution of the ring distribution for run 24 .

much distortion in the shape of the clusters. After sufficient time, however, they are all mixed and the instability saturates at the same energy level as the gyrotropic case.

When $p_{0}=601\left(>p_{C}\right)$, qualitatively speaking, the time history of the wave energy (Fig. 17) exhibits similar behavior as in the gyrotropic case (cf. Fig. 6). However, in the nongyrotropic run in which the ring electrons are initially concentrated at several phase angles, a mixture in the phase angle has to take place (due to small thermal spread in the distribution function) before the double cluster structure appears. Hence the time evolution is much slower for the nongyrotropic runs.

\section{SUMMARY AND DISCUSSION}

The long time evolution of the $k=0$ mode waves generated by the relativistic ring distribution is investigated. The dispersion relations for gyrotropic and nongyrotropic ring distributions were evaluated analytically and numerically. The nongyrotropic dispersion relation bears similar properties as those discussed by Brinca et al. ${ }^{22}$ such as the coupling of different wave modes which are independent in a gyrotropic plasma, and an enhancement of the growth rate for nongyrotropy with $m=2$.

By solving the dispersion relations with relativistic effects, it was found that there exists a critical value of the initial ring momentum, $p_{C}$, at which the system becomes

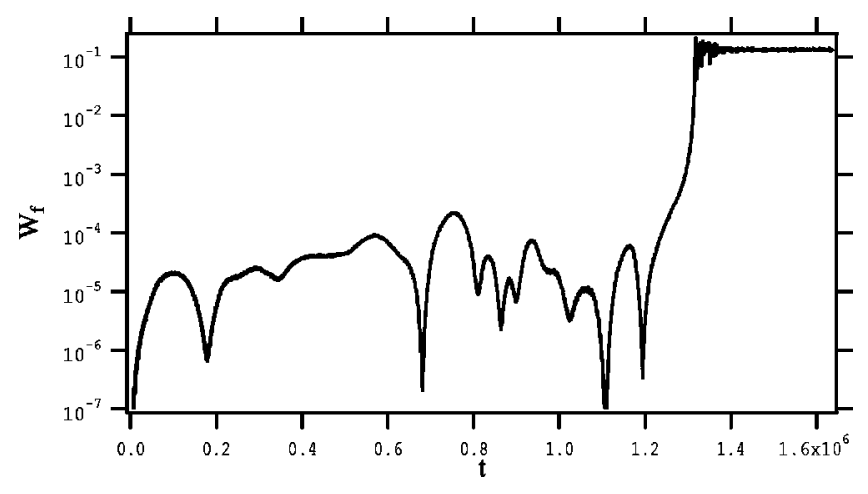

FIG. 17. The time history of the electric field energy for run 25 .

marginally stable, since the wave sustained by the background plasma can no longer resonate with the slow cyclotron motion of the ring electrons as $p_{0}$ exceeds $p_{C}$.

We performed particle simulations using various parameters and discussed the nonlinear evolution of the system. It was found that, in contrast to the prediction from the linear theory, the system can become unstable even when $p_{0}$ $>p_{C}$ : the system first settles to the double-cluster state with relatively low wave energy, and after a long time, the linear instability is triggered spontaneously due to thermal noise which is always present in the system, and the final single cluster state is realized. The saturation level of wave electric field for the single cluster state is estimated.

We have measured the transition time $\left(T_{\text {sat }}\right)$, i.e., the time required for the system to evolve from the meta-stable, double-cluster state to the eventual single-cluster state, by running several simulations giving various thermal spread to the ring electrons $\left(p_{\text {th }}\right)$. The initial average ring momentum and particle numbers are fixed at $p_{0}=800\left(>p_{C}\right)$ and $n_{e}$ $=n_{p}=n_{r}=3,000$. The results are shown in Fig. 18. When $p_{\text {th }}$ is less than 50 , the system was found to be practically stable, and the finite value of $T_{\text {sat }}$ could not be determined from the simulation. When $p_{\text {th }}$ is increased starting from 50, $T_{\text {sat }}$ gradually decreases and becomes almost constant around $55<p_{\text {th }}<65$. Then $T_{\text {sat }}$ suddenly drops as $p_{\text {th }}$ is further increased. When $p_{\text {th }}>70, T_{\text {sat }}$ decreases only slightly as $p_{\text {th }}$ is increased. This behavior is similar to the property of the Langevin model in which a mass point moves within a potential well with double local minima [Fig. 19(b)]. Initially we suppose that the mass point is located near the potential minimum to the left, which is at a higher energy level than the minimum to the right. As the amplitude of noise given to the mass point increases from zero, the transition time scale for the mass point to travel from one local minimum to the other decreases, and the time scale decreases significantly as the noise energy and the energy of the gap separating the two potential wells become equivalent. For the ring system, the condition $p_{0}>p_{C}$ corresponds to the appearance of a local minimum (the double-cluster state) at a site close to the initial state. When $p_{0}<p_{C}$, there is only one global minimum as described in Fig. 19(a). Besides the discussion above, in this paper we have almost always neglected the thermal spread of all the plasma species for simplicity. A few comments are necessary here. First, it has already been discussed 


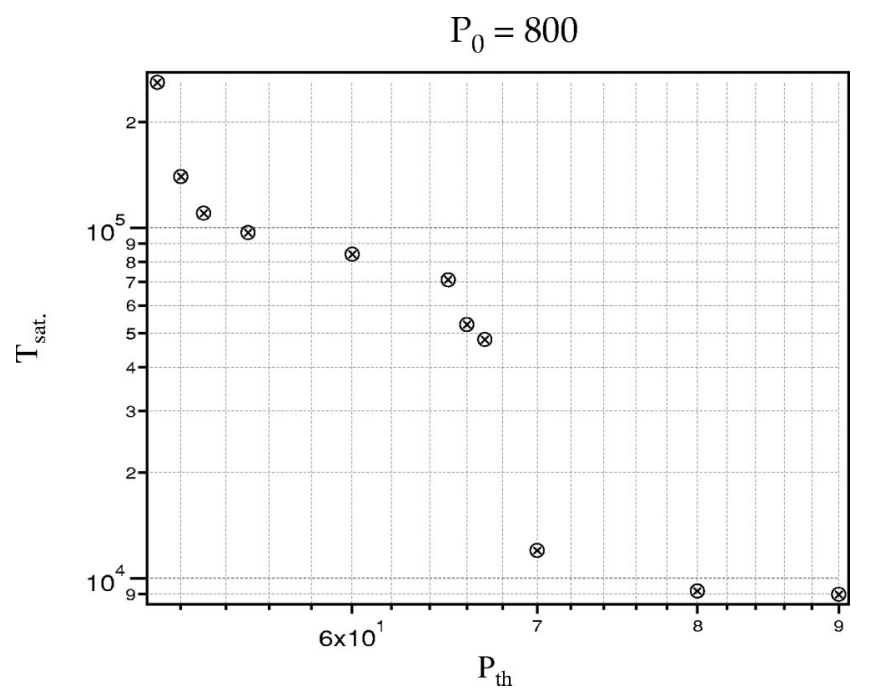

FIG. 18. The saturation time for $p_{0}=800$ as a function of the thermal spread of momentum of ring electrons.

that the thermal spread in the ring distribution allows the cyclotron damping to enter in the ring cyclotron instability, reducing the growth rate (Winglee ${ }^{8}$ ). For the $k=0$ mode also, it is natural to expect that the inclusion of the thermal spread in distribution functions modifies the linear growth rate through cyclotron resonance. However, it is unlikely that it will bring drastic qualitative changes in the time evolution of the instability. Second, in case of relativistic, nongyrotropic instability, one should pay attention to the fact that their wave frequencies have explicit dependence to the particle momentum, if the distribution function has finite thermal spread. This effect does not appear in a nonrelativistic plasma.

In realistic space and astrophysical plasma situations, the ring distribution will destabilize finite $k$ modes as well as the $k=0$ mode, and the particle free energy will be distributed among all the possible unstable wave modes. In fact, it is known that the linear growth rate for the cyclotron maser instability is maximized for perpendicularly propagating waves. We stress again that the system we studied in this paper is a special subset of space plasma in reality, with which the nonlinear time evolution can be discussed in detail.

We showed that when $p_{0}>p_{\mathrm{C}}$, the system is stable even though free energy is available. As far as the cyclotron maser instability of the fundamental resonance is concerned, this meta-stable state exists also for systems allowing finite $k$, depending on the parameters $p_{0}, a$, and $\alpha$. In a high energy astrophysical plasma, such as the pulsar driven nebulas in which $\gamma \gg 1$ and $\sigma \ll 1$, where $\sigma \equiv 1 / a \gamma$ is the Lorentz invariant ratio between the field and the particle energy, the metastable state may be realized for the fundamental resonance. However, even in this case the higher harmonic waves can be destabilized. In future publications we hope to discuss more details on the meta-stable state, including estimation of the energy levels, transition time scales, and the conditions for the meta-stable state to emerge in a more general setting of the system.

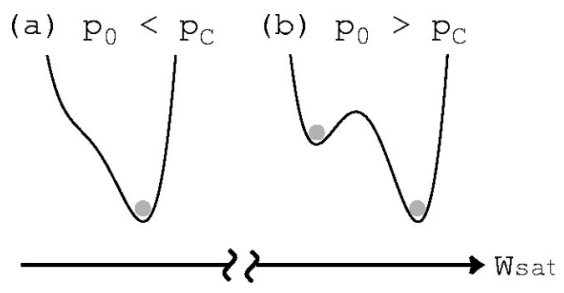

FIG. 19. The schematic picture of the potential structure.

\section{ACKNOWLEDGMENTS}

The authors sincerely thank V. V. Krasnosel'skikh, M. Nambu, and B. Lembege for valuable discussions.

This paper has been supported by JSPS Research Fellowships for Young Scientists in Japan.

\section{APPENDIX: FLUID DESCRIPTION OF THE RING DISTRIBUTION}

It is instructive to formulate the evolution of the ring distribution from the Vlasov-fluid point of view, in which the distribution is represented as a continuous ring in the momentum phase space. We assume that the ring distribution has zero width both in the parallel and perpendicular momentum directions, while the distribution is a continuous function of the phase angle (azimuthal angle) of the perpendicular momentum, $\phi$. Namely, we write the distribution function as

$$
f\left(p_{\|}, p_{\perp}\right)=N(\phi) \delta\left(p_{\|}\right) \frac{\delta\left(p_{\perp}-P(\phi)\right)}{p_{\perp}},
$$

where $P(\phi)$ is the ring momentum radius (momentum) and $N(\phi)$ is the differential density of the ring per unit azimuthal angle. By definition, $\int_{0}^{2 \pi} N(\phi) d \phi=n$ gives the ring density. The form of the distribution function defined above is valid only until the instability enters the nonlinear stage and $P(\phi)$ becomes multi-valued. Although this difficulty may be avoided by introducing parametric representations of the variables [e.g., by letting $P=P(\eta)$ and $\phi=\phi(\eta)$, with an appropriate parameter $\eta]$, we do not do so here, since our primary interest of the evolution equations derived here is in examination of the linear instability in Sec. II B.

Integrating Eq. (25) over the momentum phase space except for the azimuthal angle, one obtains the continuity equation for $N(\phi)$,

$$
\frac{\partial N(\phi)}{\partial t}-\frac{\partial}{\partial \phi}\left[N(\phi)\left(\frac{\Omega_{0}}{\gamma}+\frac{q E \sin (\phi-\eta)}{P(\phi)}\right)\right]=0,
$$

where $E$ and $\eta$ are the magnitude and the phase angle of the electric field, respectively [i.e., $\mathbf{E}=E(\cos \eta, \sin \eta)]$. The evolution of $N(\phi)$ is determined by the differential density flux in the azimuthal angle direction, which consists of the Larmor rotation [the second term in Eq. (A2)] and the acceleration of the particles due to perpendicular electric field (the third term).

The integration of Eq. (25) with a weight of $p_{\perp}$ yields the conservation of the momentum flux, 


$$
\begin{aligned}
\frac{\partial}{\partial t}( & N(\phi) P(\phi))-\frac{\partial}{\partial \phi}\left(\frac{\Omega_{0}}{\gamma} N(\phi) P(\phi)\right) \\
& =2 q E \cos (\phi-\eta) N(\phi)+q E \sin (\phi-\eta) \frac{\partial N(\phi)}{\partial \phi} .
\end{aligned}
$$

From the above two equations we find the evolution equation for $P(\phi)$,

$\frac{\partial P(\phi)}{\partial t}-\left(\frac{\Omega_{0}}{\gamma}+\frac{q E \sin (\phi-\eta)}{P(\phi)}\right) \frac{\partial P(\phi)}{\partial \phi}=q E \cos (\phi-\eta)$.

This equation indicates that the parallel electric field (RHS) determines the acceleration/deceleration of $P(\phi)$, which consists of time evolution and the convective evolution due to the Larmor rotation and due to acceleration by the perpendicular electric field.

${ }^{1}$ C. S. Wu, Space Sci. Rev. 41, 215 (1985).

${ }^{2}$ C. S. Wu and L. C. Lee, Astrophys. J. 230, 621 (1979).

${ }^{3}$ K. R. Chu and J. L. Hirshfield, Phys. Fluids 21, 461 (1978).

${ }^{4}$ R. Q. Twiss, Aust. J. Phys. 11, 564 (1958).

${ }^{5}$ J. Schneider, Phys. Rev. Lett. 2, 5044 (1959).
${ }^{6}$ A. V. Gaponov, Izv. Radiofiz. 2, 836 (1959).

${ }^{7}$ A. A. Galeev and V. V. Krasnosel'skikh, Sov. Astron. Lett. 5, 257 (1979).

${ }^{8}$ R. M. Winglee, Plasma Phys. 25, 217 (1983).

${ }^{9}$ R. A. Dory, G. E. Guest, and E. G. Harris, Phys. Rev. Lett. 14, 131 (1965).

${ }^{10}$ H. P. Freund, H. K. Wong, C. S. Wu, and M. J. Xu, Phys. Fluids 26, 2263 (1983).

${ }^{11}$ D. Le. Queau, Comput. Phys. Commun. 49, 85 (1988).

${ }^{12}$ D. Mourenas, V. V. Krasnosel'skikh, and C. Beghin, J. Geophys. Res. 98, 5855 (1993).

${ }^{13}$ P. L. Pritchett, Phys. Fluids 29, 2919 (1986).

${ }^{14}$ D. Le. Queau, R. Pellat, and A. Roux, Phys. Fluids 27, 247 (1984).

${ }^{15}$ A. S. Volokitin, V. V. Krasnosel'skikh, and G. Z. Machabeli, Sov. J. Plasma Phys. 11, 310 (1985).

${ }^{16}$ M. Hoshino and J. Arons, Phys. Fluids B 3, 818 (1991).

${ }^{17}$ A. B. Langdon, J. Arons, and C. E. Max, Phys. Rev. Lett. 61, 779 (1988).

${ }^{18}$ Y. A. Gallant, M. Hoshino, A. B. Langdon, J. Arons, and C. E. Max, Astrophys. J. 391, 73 (1992).

${ }^{19}$ M. Hoshino, J. Arons, Y. A. Gallant, and A. B. Langdon, Astrophys. J. 390, 454 (1992).

${ }^{20}$ P. Yoon and R. C. Davidson, Phys. Rev. A 35, 2619 (1987).

${ }^{21}$ R. N. Sudan, Phys. Fluids 8, 1915 (1965).

${ }^{22}$ A. L. Brinca, L. Borda de Agua, and D. Winske, J. Geophys. Res. 98, 7549 (1993).

${ }^{23}$ J. B. Cao, C. Mazelle, G. Belmont, and H. Reme, J. Geophys. Res. 100, 23379 (1995).

${ }^{24}$ H. P. Freund, J. Q. Dong, C. S. Wu, and L. C. Lee, Phys. Fluids 30, 3106 (1987). 